\title{
Volume Cauchy formulas for slice functions on real associative ${ }^{*}$-algebras
}

\author{
R. Ghiloni, A. Perotti * \\ Department of Mathematics \\ University of Trento \\ Via Sommarive, 14 \\ I-38123 Povo Trento ITALY \\ ghiloni@science.unitn.it, perotti@science.unitn.it
}

\begin{abstract}
We introduce a family of Cauchy integral formulas for slice and slice regular functions on a real associative *-algebra. For every suitable choice of a real subspace of the algebra, a different formula is given, in which the domains of integration are subsets of the subspace. In particular, in the quaternionic case we get a volume Cauchy formula. In the Clifford algebra case, the choice of the paravector subspace $\mathbb{R}^{n+1}$ gives a volume Cauchy formula for slice monogenic functions.
\end{abstract}

Keywords: Cauchy integral formula; slice regular function; quaternions; Clifford algebras

AMS Subject Classification: Primary 30G35; Secondary 32A30, 30E20, 13J30

\section{Introduction}

The concept of slice regularity for functions of one quaternionic, octonionic or Clifford variable has been introduced recently by Gentili and Struppa [1, 2] and by Colombo, Sabadini and Struppa [3]. In [4] and [5], a new approach to slice functions, based on the concept of stem function, allowed to extend further the theory to any real alternative *algebra of finite dimension. In this setting, a Cauchy integral formula for slice functions of class $\mathscr{C}^{1}$ was proved. In the quaternionic case, a Cauchy kernel was already introduced in [6], and for slice monogenic functions in $[7,8]$. This kernel was applied in [9] to get Cauchy formulas for $\mathscr{C}^{1}$-functions on a class of domains intersecting the real axis.

In the present work, we introduce a family of Cauchy integral formulas for slice and slice regular functions, in which the domains of integration depend on the choice of a suitable real vector subspace of the algebra. In the quaternionic case, taking as subspace the whole space $\mathbb{H}$, we get a volume Cauchy formula. In the Clifford algebra case, we can

\footnotetext{
*Work partially supported by GNSAGA of INdAM
} 
choose the paravector subspace $\mathbb{R}^{n+1}$, obtaining a Cauchy formula in which integration is performed on an open subset of $\mathbb{R}^{n+1}$ and on its boundary.

We begin by fixing some assumptions and by recalling some basic notions.

Fix a real associative algebra $A$ with unity 1 of finite dimension $d>0$, equipped with the $\mathscr{C}^{\infty}$-manifold structure as a real vector space and with an anti-involution $x \longmapsto x^{c}$. Define $e:=d-1$. Identify $\mathbb{R}$ with the subalgebra of $A$ generated by 1 . The antiinvolution $x \longmapsto x^{c}$ is a real linear map of $A$ into $A$ satisfying the following properties: $\left(x^{c}\right)^{c}=x$ for all $x \in A,(x y)^{c}=y^{c} x^{c}$ for all $x, y \in A$ and $x^{c}=x$ for each real $x$. We can then consider $A$ as a real ${ }^{*}$-algebra.

For each element $x$ of $A$, the trace of $x$ is $t(x):=x+x^{c} \in A$ and the (squared) norm of $x$ is $n(x):=x x^{c} \in A$. We recall some definitions from [4] and [5].

Definition 1.1. The quadratic cone of $A$ is the set

$$
\mathcal{Q}_{A}:=\mathbb{R} \cup\left\{x \in A \mid t(x) \in \mathbb{R}, n(x) \in \mathbb{R}, 4 n(x)>t(x)^{2}\right\} .
$$

We also set $\mathbb{S}_{A}:=\left\{J \in \mathcal{Q}_{A} \mid J^{2}=-1\right\}$. Elements of $\mathbb{S}_{A}$ are called square roots of -1 in the algebra $A$. For each $J \in \mathbb{S}_{A}$, we will denote by $\mathbb{C}_{J}:=\langle 1, J\rangle \simeq \mathbb{C}$ the subalgebra of A generated by $J$.

In what follows, we assume that $\mathbb{S}_{A} \neq \emptyset$. It follows that $d$ is even.

Let $A_{\mathbb{C}}=A \otimes_{\mathbb{R}} \mathbb{C}$ be the complexification of $A$. We will use the representation $A_{\mathbb{C}}=\{w=x+i y \mid x, y \in A\}$, with $i^{2}=-1$ and complex conjugation $\bar{w}=\overline{x+i y}=x-i y$.

Let $D$ be a non-empty subset of $\mathbb{C}$, invariant under the complex conjugation $z=$ $\alpha+i \beta \longmapsto \bar{z}=\alpha-i \beta$. A function $F: D \longrightarrow A_{\mathbb{C}}$ is called a stem function on $D$ if it satisfies the condition $F(\bar{z})=\overline{F(z)}$ for each $z \in D$. If $F_{1}, F_{2}: D \longrightarrow A$ are the $A$-valued components of $F=F_{1}+i F_{2}$, then such a condition is equivalent to require that $F_{1}(\bar{z})=F_{1}(z)$ and $F_{2}(\bar{z})=-F_{2}(z)$ for each $z \in D$. We call $F$ continuous if $F_{1}$ and $F_{2}$ are continuous. We say that $F$ is of class $\mathscr{C}^{1}$ if $F_{1}$ and $F_{2}$ can be extended on an open neighborhood $U$ of $D$ in $\mathbb{C}$ to functions $\tilde{F}_{1}, \tilde{F}_{2}$ of class $\mathscr{C}^{1}$ in the usual sense. The reader observes that one can also suppose that $U$ is invariant under the complex conjugation of $\mathbb{C}$ and $\tilde{F}_{1}+i \tilde{F}_{2}$ is a stem function.

Let $\Omega_{D}$ be the subset of $\mathcal{Q}_{A}$ defined by:

$$
\Omega_{D}:=\left\{x \in \mathcal{Q}_{A} \mid x=\alpha+\beta J, \alpha, \beta \in \mathbb{R}, \alpha+i \beta \in D, J \in \mathbb{S}_{A}\right\} .
$$

We set $D_{J}:=\Omega_{D} \cap \mathbb{C}_{J}$ and denote by $\partial D_{J}$ the (relative topological) boundary of $D_{J}$ in $\mathbb{C}_{J}$. Observe that, if $D$ is open, then $\Omega_{D}$ is relatively open in $\mathcal{Q}_{A}$ and the boundary $\partial \Omega_{D}$ of $\Omega_{D}$ in $\mathcal{Q}_{A}$ coincides with $\bigcup_{J \in \mathbb{S}_{A}} \partial D_{J}$.

Definition 1.2. Any stem function $F=F_{1}+i F_{2}: D \longrightarrow A_{\mathbb{C}}$ induces a (left) slice function $f=\mathcal{I}(F): \Omega_{D} \longrightarrow A$ as follows: if $x=\alpha+\beta J \in D_{J}$ for some $\alpha, \beta \in \mathbb{R}$ and $J \in \mathbb{S}_{A}$, we set

$$
f(x):=F_{1}(z)+J F_{2}(z) \quad(z=\alpha+i \beta) .
$$


We will denote by $\mathcal{S}^{0}\left(\Omega_{D}, A\right)$ the real vector space of (left) slice functions on $\Omega_{D}$ induced by continuous stem functions and by $\mathcal{S}^{1}\left(\Omega_{D}, A\right)$ the real vector space of slice functions induced by stem functions of class $\mathscr{C}^{1}$. Proposition 7 of [4] ensures that $\mathcal{S}^{h}\left(\Omega_{D}, A\right) \subset \mathscr{C}^{0}\left(\Omega_{D}, A\right)(h=0,1)$.

Suppose that $D$ is a non-empty open subset of $\mathbb{C}$. Let $F: D \longrightarrow A_{\mathbb{C}}$ be a stem function of class $\mathscr{C}^{1}$ and let $f=\mathcal{I}(F) \in \mathcal{S}^{1}\left(\Omega_{D}, A\right)$. Let us denote by $\partial F / \partial \bar{z}: D \longrightarrow A_{\mathbb{C}}$ the stem function on $D$ defined by

$$
\frac{\partial F}{\partial \bar{z}}:=\frac{1}{2}\left(\frac{\partial F}{\partial \alpha}+i \frac{\partial F}{\partial \beta}\right)
$$

which induces the slice derivative $\frac{\partial f}{\partial x^{c}}:=\mathcal{I}\left(\frac{\partial F}{\partial \bar{z}}\right) \in \mathcal{S}^{0}\left(\Omega_{D}, A\right)$.

Definition 1.3. A slice function $f \in \mathcal{S}^{1}\left(\Omega_{D}, A\right)$ is called slice regular if it holds:

$$
\frac{\partial f}{\partial x^{c}}=0 \quad \text { on } \Omega_{D} \text {. }
$$

We denote by $\mathcal{S R}\left(\Omega_{D}, A\right)$ the real vector space of all slice regular functions on $\Omega_{D}$.

Let $S$ be a non-empty subset of $A$. We say that $S$ is a genuine imaginary sphere of $A$, for short a gis of $A$, if there exists a (real) vector subspace $M$ of $A$ such that $\mathbb{R} \subset M \subset \mathcal{Q}_{A}$ and $S=M \cap \mathbb{S}_{A}$. If such a $M$ exists, then it is unique. In fact, it is easy to verify that $M=\bigcup_{J \in S} \mathbb{C}_{J}$. For this reason, if $S$ is a gis of $A$, then we say that $M$ is the vector subspace of $A$ inducing $S$. The reader observes that, since $M$ contains 1 and at least one element of $\mathbb{S}_{A}$, its dimension is at least 2. Moreover, the set $\{J,-J\}$ is a gis of $A$ for each $J \in \mathbb{S}_{A}$.

Lemma 1.4. Let $S$ be a gis of $A$ and let $M$ be the vector subspace of $A$ inducing $S$. Then there exists a norm \|\| on $A$ such that $\|x\|^{2}=n(x)$ for each $x \in M$.

Proof. Since $M \subset \mathcal{Q}_{A}$, the function $n(x)$ is real-valued and non-negative on $M$. We prove that the function $\sqrt{n(x)}$ is a norm on $M$. First of all, the function is positivehomogeneous and vanishes only at $x=0$. It remains to prove that it satisfies the triangle inequality:

$$
\sqrt{n(x+y)} \leq \sqrt{n(x)}+\sqrt{n(y)} \text { for each } x, y \in M
$$

This is equivalent to

$$
n(x+y)-n(x)-n(y) \leq 2 \sqrt{n(x) n(y)} .
$$

The left-hand side of (1) is $x y^{c}+y x^{c}=t\left(x y^{c}\right)$. Let $x=\alpha+\beta I, y=\alpha^{\prime}+\beta^{\prime} J$ in $M$, with $I, J \in S \subset \mathbb{S}_{A}, \alpha, \beta, \alpha^{\prime}, \beta^{\prime} \in \mathbb{R}$ and $\beta, \beta^{\prime} \geq 0$. Since $I-J \in M$, we have that $n(I-J) \in \mathbb{R}$ and

$$
0 \leq n(I-J)=-(I-J)^{2}=2+t(I J) .
$$

It follows that $t(I J)$ is real and $t(I J) \geq-2$. Since $x y^{c}=(\alpha+\beta I)\left(\alpha^{\prime}-\beta^{\prime} J\right)$, the trace of $x y^{c}$ is equal to $2 \alpha \alpha^{\prime}-\beta \beta^{\prime} t(I J)$. Therefore, $t\left(x y^{c}\right) \leq 2\left(\alpha \alpha^{\prime}+\beta \beta^{\prime}\right)$, while $n(x) n(y)=\left(\alpha^{2}+\beta^{2}\right)\left(\alpha^{\prime 2}+\beta^{\prime 2}\right) \geq\left(\alpha \alpha^{\prime}+\beta \beta^{\prime}\right)^{2}$. It follows that $t\left(x y^{c}\right) \leq 2 \sqrt{n(x) n(y)}$, which is precisely inequality (1). Since the restriction of $\sqrt{n(x)}$ to $M$ is a norm, it can be extended to a norm \|\| on $A$. 


\section{The volume Cauchy formulas}

Fix a gis $S$ of $A$. Denote by $M$ the vector subspace of $A$ inducing $S$ and by $m$ the dimension of $M$. Choose a norm \|\| on $A$ as in Lemma 1.4.

Let $\mathcal{B}=\left(v_{0}, v_{1}, \ldots, v_{e}\right)$ be a (real) vector basis of $A$ with $v_{0}=1$, orthonormal w.r.t. the scalar product $($, ) on $A$ associated to the norm \|\| and such that $v=$ $\left(v_{0}, v_{1}, \ldots, v_{m-1}\right)$ form a orthonormal basis of $M$. Note that $M$ is the orthogonal direct sum of $\mathbb{R}$ and $M \cap \operatorname{ker}(t)$, since $(x, y)=\frac{1}{2} t\left(x y^{c}\right)$ on $M$. Let $L: \mathbb{R}^{d} \longrightarrow A$ be the real vector isomorphism sending $x=\left(x_{0}, x_{1}, \ldots, x_{e}\right)$ into $L(x)=\sum_{\ell=0}^{e} x_{\ell} v_{\ell}$. Identify $\mathbb{R}^{d}$ with $A$ via $L$ and hence $M$ with $\mathbb{R}^{m}=\mathbb{R}^{m} \times\{0\} \subset \mathbb{R}^{m} \times \mathbb{R}^{d-m}=\mathbb{R}^{d}$. The product of $A$ becomes a product on $\mathbb{R}^{d}$ by requiring that $L$ is an isomorphism of $\mathbb{R}$-algebras. In other words, given $x, y \in \mathbb{R}^{d}, x y$ is defined as $L^{-1}(L(x) L(y))$. Since $\mathcal{B}$ is orthonormal, $\|x\|$ coincides with the usual euclidean norm $\left(\sum_{\ell=0}^{e} x_{\ell}^{2}\right)^{1 / 2}$ of $x$ in $\mathbb{R}^{d}$. By Proposition $1(6)$ of [4], we know that $\mathbb{S}_{A}=\{J \in A \mid t(J)=0, n(J)=1\}$. In this way, we have that

$$
S=\left\{\left(x_{0}, x_{1}, \ldots, x_{m-1}\right) \in \mathbb{R}^{m} \mid x_{0}=0, \sum_{\ell=1}^{m-1} x_{\ell}^{2}=1\right\} .
$$

In what follows, we assume that $D$ is a non-empty bounded open subset of $\mathbb{C}$ with boundary $\partial D$ of class $\mathscr{C}^{1}$. Denote by $\mathrm{n}: \partial D \longrightarrow \mathbb{C}$ the continuous function sending $z \in \partial D$ into the outer normal versor of $\partial D$ at $z$. Since $D$ is stable under conjugation, the map $\mathrm{n}$ is a stem function on $\partial D$.

We define the circularization $\Omega_{D}(S)$ of $D$ w.r.t. $S$ as the following subset of $\mathcal{Q}_{A}$ :

$$
\Omega_{D}(S):=\left\{x \in \mathcal{Q}_{A} \mid x=\alpha+\beta J, \alpha, \beta \in \mathbb{R}, \alpha+i \beta \in D, J \in S\right\} .
$$

Since $\Omega_{D}(\{J,-J\})=D_{J}$ for each $J \in \mathbb{S}_{A}$, it follows that $\Omega_{D}(S)=\bigcup_{J \in S} D_{J}$. On the other hand, $M=\bigcup_{J \in S} \mathbb{C}_{J}$ and hence $\Omega_{D}(S)$ is an open subset of $M$. Denote by $\partial \Omega_{D}(S)$ the boundary of $\Omega_{D}(S)$ in $M$. It is easy to see that $\partial \Omega_{D}(S)=\bigcup_{J \in S} \partial D_{J}=\Omega_{\partial D}(S)$. We define the outer normal vector field $\mathbf{n}: \partial \Omega_{D}(S) \longrightarrow A$ to $\partial \Omega_{D}(S)$ as the slice function induced by the stem function $\mathbf{n}$. More explicitly, $\mathbf{n}$ is defined as follows. Given $x=\alpha+\beta J \in \partial \Omega_{D}(S), z:=\alpha+i \beta$ belongs to $\partial D$ and hence we can write $\mathrm{n}(z)=\mathrm{n}_{1}+i \mathrm{n}_{2}$ for some $\mathrm{n}_{1}, \mathrm{n}_{2} \in \mathbb{R}$. Then we have: $\mathbf{n}(x):=\mathrm{n}_{1}+\mathrm{n}_{2} J$.

For each non-negative integer $n$, we denote by $\eta_{n}$ the volume of the standard sphere $S^{n}$ of $\mathbb{R}^{n+1}$. It is well known that $\eta_{n}$ has the following explicit expression:

$$
\eta_{n}=\frac{2 \pi^{\frac{n+1}{2}}}{\Gamma\left(\frac{n+1}{2}\right)},
$$

where $\Gamma$ is Euler's gamma function.

Let us introduce a notion of Cauchy kernel of $A$ relative to the gis $S$.

We start recalling from [4] the definitions of the characteristic polynomial $\Delta_{w}$ of $w \in \mathcal{Q}_{A}$ and of the Cauchy kernel of $A . \Delta_{w}$ is the slice regular polynomial

$$
\Delta_{w}(x):=x^{2}-x t(w)+n(w),
$$


with zero set $\mathbb{S}_{w}:=\left\{x \in \mathcal{Q}_{A} \mid t(x)=t(w), n(x)=n(w)\right\}$. The Cauchy kernel for slice regular functions on $A$ is defined, for each $x \in \mathcal{Q}_{A} \backslash \mathbb{S}_{w}$, as

$$
C(x, w):=\Delta_{w}(x)^{-1}\left(w^{c}-x\right) .
$$

$C(\cdot, w)$ is slice regular on $\mathcal{Q}_{A} \backslash \mathbb{S}_{w}$ and has the following property w.r.t. the slice product of functions:

$$
C(x, w) \cdot(w-x)=1 .
$$

Define $\Gamma_{S}:=\left\{(x, w) \in \mathcal{Q}_{A} \times(M \backslash \mathbb{R}) \mid \Delta_{w}(x) \neq 0\right\}$. Observe that, if $(x, w) \in \Gamma_{S}$ and $x=\alpha+\beta J$ for some $\alpha, \beta \in \mathbb{R}$ and $J \in S$, then $\Delta_{w}(x)$ belongs to $\mathbb{C}_{J} \backslash\{0\}$ and hence $\Delta_{w}(x)$ is invertible in $A$, or better, in $\mathbb{C}_{J}$. This fact ensures that the following definition is consistent.

Definition 2.1. We define the Cauchy kernel of $A$ w.r.t. $S$ as the smooth function $C_{S}: \Gamma_{S} \longrightarrow A$ given by setting

$$
C_{S}(x, w):=\frac{2}{\eta_{m-2}} \frac{C(x, w)}{(n(\operatorname{Im}(w)))^{\frac{m-2}{2}}}=\frac{2}{\eta_{m-2}} \frac{C(x, w)}{\|\operatorname{Im}(w)\|^{m-2}},
$$

where $\operatorname{Im}(w):=\left(w-w^{c}\right) / 2$.

Note that, for each fixed $w \in M \backslash \mathbb{R}, C_{S}(\cdot, w)$ is a slice regular function on $\mathcal{Q}_{A} \backslash \mathbb{S}_{w}$. In particular, when $S=\{J,-J\}$ for some $J \in \mathbb{S}_{A}$, then $m=2, \eta_{m-2}=2$, and $C_{S}$ extends up to the real axis and coincides with the kernel $C$ of $A$.

Denote by $w=\left(w_{0}, w_{1}, \ldots, w_{m-1}\right): M \longrightarrow \mathbb{R}^{m}$ the coordinate system on $M$ sending $w$ into $\sum_{\ell=0}^{m-1} w_{\ell} v_{\ell}$ and by $d w$ the corresponding volume form $d w_{0} \wedge d w_{1} \wedge \cdots \wedge d w_{m-1}$. Such a volume form induces a structure of oriented Riemannian manifold on $M$ and hence on its open subset $\Omega_{D}(S)$. Its boundary $\partial \Omega_{D}(S)$ is a hypersurface of $M$ of class $\mathscr{C}^{1}$, which inherits a structure of oriented Riemannian manifold, via the standard rule "first the outer normal vector". Denote by $d \sigma_{w}$ the corresponding volume form of $\partial \Omega_{D}(S)$. By abusing notation, we will use the letter $w$ to indicate both a point of $M$ and its coordinates w.r.t. $v$.

We have:

Lemma 2.2. For each $x \in \Omega_{D}(S)$, the function from $\Omega_{D}(S) \backslash\left(\mathbb{S}_{x} \cup \mathbb{R}\right)$ to $A$, sending $w$ into $C_{S}(x, w)$, is summable on $\Omega_{D}(S)$ w.r.t. $d w$ and the function from $\partial \Omega_{D}(S) \backslash \mathbb{R}$ to $A$, sending $w$ into $C_{S}(x, w)$, is summable on $\partial \Omega_{D}(S)$ w.r.t. $d \sigma_{w}$.

Denote by $\bar{\Omega}_{D}$ the closure of $\Omega_{D}$ in $\mathcal{Q}_{A}$, which coincides with $\Omega_{\bar{D}}$ if $\bar{D}$ denotes the closure of $D$ in $\mathbb{C}$.

We are now in position to state our main result.

Theorem 2.3. Let $S$ be a gis of $A$, let $M$ be the vector subspace of $A$ inducing $S$ and let $m:=\operatorname{dim} M$. Choose a volume form $d w$ of $M$ as above and denote by $d \sigma_{w}$ the 
corresponding volume form of $\partial \Omega_{D}(S)$. Then, for each $f \in \mathcal{S}^{1}\left(\bar{\Omega}_{D}, A\right)$ and for each $x \in \Omega_{D}$, it holds:

$$
f(x)=\frac{1}{2 \pi} \int_{\partial \Omega_{D}(S)} C_{S}(x, w) \mathbf{n}(w) f(w) d \sigma_{w}-\frac{1}{\pi} \int_{\Omega_{D}(S)} C_{S}(x, w) \frac{\partial f}{\partial x^{c}}(w) d w .
$$

If $f$ is slice regular on $\Omega_{D}$, then formula (2) holds with only the boundary term.

Formula (2) is still valid for all functions in $\mathcal{S}^{1}\left(\Omega_{D}, A\right)$, which admit a continuous extension on $\bar{\Omega}_{D}$. This can be seen by approximating the domain with smaller subdomains. Using the same strategy, one can relax also the assumption on the $\mathscr{C}^{1}$-regularity of $\partial D$ and hence of $\partial \Omega_{D}(S)$.

The reader observes that, if $S$ is equal to the gis $\{J,-J\}$ for some $J \in \mathbb{S}_{A}$, then formula (2) reduces to the Cauchy formula obtained in Theorem 27 of [4].

For each continuous slice function $f \in \mathcal{S}^{0}\left(\partial \Omega_{D}(S), A\right)$, we can consider the Cauchytype integrals $F_{S}^{+}: \Omega_{D} \longrightarrow A$ and $F_{S}^{-}: \mathcal{Q}_{A} \backslash \bar{\Omega}_{D} \longrightarrow A$ defined respectively by setting

$$
F_{S}^{ \pm}(x):=\frac{1}{2 \pi} \int_{\partial \Omega_{D}(S)} C_{S}(x, w) \mathbf{n}(w) f(w) d \sigma_{w} .
$$

Observe that $F_{S}^{ \pm}$are slice regular functions. If the boundary function $f$ is of class $\mathscr{C}^{1}$ (in fact, a Hölder condition suffices), then a Sokhotskiü-Plemelj jump formula is valid.

Theorem 2.4. Let $S$ be a gis of $A$, let $f \in \mathcal{S}^{1}\left(\partial \Omega_{D}(S), A\right)$ and let $F_{S}^{ \pm}$be the functions defined in (3). Then $F_{S}^{+}$extends continuously to $\bar{\Omega}_{D}$ and $F_{S}^{-}$extends continuously to $\mathcal{Q}_{A} \backslash \Omega_{D}$. Moreover, for each $x \in \partial \Omega_{D}(S)$, it holds:

$$
f(x)=F_{S}^{+}(x)-F_{S}^{-}(x) .
$$

Corollary 2.5. Let $S$ be a gis of $A$ and let $f \in \mathcal{S}^{1}\left(\partial \Omega_{D}(S), A\right)$. Then there exists $F \in \mathcal{S R}\left(\Omega_{D}, A\right) \cap \mathscr{C}^{0}\left(\bar{\Omega}_{D}, A\right)$ such that $F=f$ on $\partial \Omega_{D}(S)$ if and only if $F_{S}^{-}$vanishes on $\partial \Omega_{D}(S)$. In this case, the extension $F$ is given by $F_{S}^{+}$.

Remark 2.6. In general, the integrals $F_{S}^{ \pm}$depend on $S$. For example, if $A=\mathbb{H}, \Omega_{D}$ is the unit ball, $S=\{i,-i\}, S^{\prime}=\{j,-j\}$ and $f(x)=x_{0}+i x_{1}$, then $F_{S}^{+}(x)=x, F_{S}^{-}(x)=0$, while $F_{S^{\prime}}^{+}(x)=x / 2, F_{S^{\prime}}^{-}(x)=-1 /(2 x)$.

We give the proof of the preceding results in the next section.

We conclude the present section by reformulating our Cauchy formula (2) in the quaternionic and in the Clifford algebra cases.

\subsection{The quaternionic case}

If $A$ is the algebra $\mathbb{H}$ of (real) quaternions, the quadratic cone is the whole algebra (see [4]) and therefore we can take $M=\mathbb{H}$ and $S=\mathbb{S}_{\mathbb{H}}$ as a gis. In this case, $\Omega_{D}(S)=\Omega_{D}$ is an open domain in $\mathbb{H}$, with $\partial \Omega_{D}(S)=\partial \Omega_{D}$. 
Corollary 2.7. For each slice function $f \in \mathcal{S}^{1}\left(\bar{\Omega}_{D}, \mathbb{H}\right)$ and for each $x \in \Omega_{D}$, it holds:

$$
f(x)=\frac{1}{2 \pi} \int_{\partial \Omega_{D}} C_{\mathbb{S}_{\mathbb{H}}}(x, w) \mathbf{n}(w) f(w) d \sigma_{w}-\frac{1}{\pi} \int_{\Omega_{D}} C_{\mathbb{S}_{\mathbb{H}}}(x, w) \frac{\partial f}{\partial x^{c}}(w) d w,
$$

where, for $x \in \mathbb{H}$ and $w=w_{0}+w_{1} i+w_{2} j+w_{3} k \in \mathbb{H} \backslash \mathbb{R}$ with $w \notin \mathbb{S}_{x}$, the kernel is

$$
C_{\mathbb{S}_{\mathbb{H}}}(x, w)=\frac{1}{2 \pi} \frac{\Delta_{w}(x)^{-1}(\bar{w}-x)}{w_{1}^{2}+w_{2}^{2}+w_{3}^{2}} .
$$

\subsection{The Clifford algebra case}

If $A$ is the real Clifford algebra $\mathbb{R}_{n}$ with signature $(0, n)$, the quadratic cone $\mathcal{Q}_{n}$ contains the subspace $\mathbb{R}^{n+1}$ of paravectors (see $[4,5]$ ). We can then take $M=\mathbb{R}^{n+1}$ and $S=$ $\mathbb{S}^{n-1}=\left\{x=x_{1} e_{1}+\cdots+x_{n} e_{n} \in \mathbb{R}_{n} \mid x_{1}^{2}+\cdots+x_{n}^{2}=1\right\}$ as a gis. Here $e_{1}, \ldots, e_{n}$ denote the basic generators of $\mathbb{R}_{n}$, satisfying the relations $e_{i} e_{j}+e_{j} e_{i}=-2 \delta_{i j}$. In this case, $\Omega_{D}\left(\mathbb{S}^{n-1}\right)$ is an open domain in $\mathbb{R}^{n+1}$ and we can take $v=\left(1, e_{1}, \ldots, e_{n}\right)$ as orthonormal basis of $M$. Slice regularity on $\mathbb{R}_{n}$ generalizes the concept of slice monogenic functions introduced in [3]. If $D$ intersects the real axis, then the restriction of a slice regular function $f$ on $\Omega_{D}$ to $\mathbb{R}^{n+1}$ is a slice monogenic function. Conversely, each slice monogenic function is the restriction of a unique slice regular function.

Corollary 2.8. Let $w=w_{0}+w_{1} e_{1}+\cdots+w_{n} e_{n} \in \mathbb{R}^{n+1} \backslash \mathbb{R}$ and let $\bar{w}=w_{0}-w_{1} e_{1}-$ $\cdots-w_{n} e_{n}$ be its Clifford conjugate. For $x \in \mathcal{Q}_{\mathbb{R}_{n}}$ and $w \notin \mathbb{S}_{x}$, consider the Cauchy kernel

$$
C_{\mathbb{S}^{n-1}}(x, w)=\frac{2}{\eta_{n-1}} \frac{\Delta_{w}(x)^{-1}(\bar{w}-x)}{\left(w_{1}^{2}+\cdots+w_{n}^{2}\right)^{\frac{n-1}{2}}} .
$$

Then, for each slice function $f \in \mathcal{S}^{1}\left(\bar{\Omega}_{D}, \mathbb{R}_{n}\right)$ and for each $x \in \Omega_{D}$, it holds:

$$
f(x)=\frac{1}{2 \pi} \int_{\partial \Omega_{D}\left(\mathbb{S}^{n-1}\right)} C_{\mathbb{S}^{n-1}}(x, w) \mathbf{n}(w) f(w) d \sigma_{w}-\frac{1}{\pi} \int_{\Omega_{D}\left(\mathbb{S}^{n-1}\right)} C_{\mathbb{S}^{n-1}}(x, w) \frac{\partial f}{\partial x^{c}}(w) d w .
$$

If $f$ is slice monogenic on $\Omega_{D}\left(\mathbb{S}^{n-1}\right)$, continuous up to the boundary, and $x \in \Omega_{D}\left(\mathbb{S}^{n-1}\right)$, then

$$
f(x)=\frac{1}{2 \pi} \int_{\partial \Omega_{D}\left(\mathbb{S}^{n-1}\right)} C_{\mathbb{S}^{n-1}}(x, w) \mathbf{n}(w) f(w) d \sigma_{w} .
$$

Another instance of Cauchy formula (2) on $\mathbb{R}_{n}$ can be obtained by choosing the gis $S=\{J,-J\}$ for some imaginary unit $J \in \mathbb{S}_{\mathbb{R}_{n}}$. If $J \in \mathbb{S}^{n-1} \subset \mathbb{S}_{\mathbb{R}_{n}}$, then formula (2) reduces to the Cauchy and Pompeiu formulas given in $[7,9]$ for slice monogenic functions.

\section{Proofs}

Let us construct explicitly polar coordinates on the standard sphere $S^{n}$ of $\mathbb{R}^{n+1}$ and on $\mathbb{R}^{n+1}$ itself. 
Let $I_{1}$ be the interval $(0,2 \pi)$ of $\mathbb{R}$, let $I_{1}^{+}$be the interval $(0, \pi)$, let $N$ be the subset $[0,+\infty) \times\{0\}$ of $\mathbb{R}^{2}=\mathbb{R} \times \mathbb{R}$ and let $\varphi_{1}: I_{1} \longrightarrow \mathbb{R}^{2} \backslash N$ be the smooth embedding defined by setting $\varphi_{1}\left(\theta_{1}\right):=\left(\cos \left(\theta_{1}\right), \sin \left(\theta_{1}\right)\right)^{T}$. For each $n \geq 2$, identify $\mathbb{R}^{n}$ with $\mathbb{R}^{n-1} \times \mathbb{R}$ and define the open subsets $I_{n}$ and $I_{n}^{+}$of $\mathbb{R}^{n}$ and the smooth embedding $\varphi_{n}: I_{n} \longrightarrow \mathbb{R}^{n+1} \backslash\left(N \times \mathbb{R}^{n-2}\right)$ by induction as follows: $I_{n}:=I_{n-1} \times(-\pi / 2, \pi / 2)$, $I_{n}^{+}:=I_{n-1} \times(0, \pi / 2)$ and $\varphi_{n}\left(\theta^{\prime}, \theta_{n}\right):=\left(\cos \left(\theta_{n}\right) \varphi_{n-1}\left(\theta^{\prime}\right), \sin \left(\theta_{n}\right)\right)^{T}$.

Let $n \geq 1$. It is easy to verify that $\varphi_{n}\left(I_{n}\right)$ is equal to the dense open subset $S^{n} \backslash\left(N \times \mathbb{R}^{n-1}\right)$ of $S^{n}$, where $N \times \mathbb{R}^{n-1}$ denotes $N$ if $n=1$. The map $\varphi_{n}$ is a smooth diffeomorphism onto its image, called polar coordinates on $S^{n}$. Similarly, if $S_{+}^{n}$ denotes the northern hemisphere $S^{n} \cap\left\{x_{n+1}>0\right\}$ of $S^{n}$, then $\varphi_{n}$ induces a smooth diffeomorphism from $I_{n}^{+}$to the dense open subset $S_{+}^{n} \backslash\left(N \times \mathbb{R}^{n-1}\right)$ of $S_{+}^{n}$.

Let $\mathbb{R}_{*}:=\mathbb{R} \backslash\{0\}$. Define $H_{n+1}:=\left(N \times \mathbb{R}^{n-1}\right) \cup\left(\mathbb{R}^{n} \times\{0\}\right) \subset \mathbb{R}^{n+1}$ and the polar coordinates $\Phi_{n+1}: \mathbb{R}_{*} \times I_{n}^{+} \longrightarrow \mathbb{R}^{n+1} \backslash H_{n+1}$ of $\mathbb{R}^{n+1}$ by setting $\Phi_{n+1}(\rho, \theta):=\rho \varphi_{n}(\theta)$. Denote by $J_{\Phi_{n+1}}$ and $J_{\varphi_{n}}$ the jacobian matrices of $\Phi_{n+1}$ and of $\varphi_{n}$, respectively. Observe that, given $\rho \in \mathbb{R}_{*}$ and $\theta \in I_{n}^{+}, J_{\Phi_{n+1}}(\rho, \theta)$ is equal to the block matrix $\left(\varphi_{n}(\theta) \mid \rho J_{\varphi_{n}}(\theta)\right)$. Define the smooth function $\mathscr{I}_{n}: I_{n}^{+} \longrightarrow \mathbb{R}$ by setting

$$
\mathscr{I}_{n}(\theta):=\operatorname{det}\left(J_{\Phi_{n+1}}(1, \theta)\right)=\operatorname{det}\left(\left(\varphi_{n}(\theta) \mid J_{\varphi_{n}}(\theta)\right)\right)
$$

for each $\theta \in I_{n}^{+}$. Let us prove an elementary, but very useful, lemma.

Lemma 3.1. For each integer $n \geq 1$, the following assertions hold:

(i) $\operatorname{det}\left(J_{\Phi_{n+1}}(\rho, \theta)\right)=\rho^{n} \mathscr{I}_{n}(\theta)$ for each $(\rho, \theta) \in \mathbb{R}_{*} \times I_{n}^{+}$.

(ii) $\left(\varphi_{n}\right)^{T} J_{\varphi_{n}}=0$ on $I_{n}^{+}$.

(iii) $\mathscr{I}_{n}(\theta)=\prod_{k=2}^{n}\left(\cos \left(\theta_{k}\right)\right)^{k-1}$ for each $\theta=\left(\theta_{1}, \ldots, \theta_{n}\right) \in I_{n}^{+}$. The latter product reduces to 1 if $n=1$.

(iv) $\mathscr{I}_{n}=\operatorname{det}\left(\left(\varphi_{n} \mid J_{\varphi_{n}}\right)\right)>0$ on $I_{n}^{+}$.

(v) $\mathscr{I}_{n}=\sqrt{\operatorname{det}\left(\left(J_{\varphi_{n}}\right)^{T} J_{\varphi_{n}}\right)}$ on $I_{n}^{+}$.

(vi) $\int_{I_{n}^{+}} \mathscr{I}_{n}(\theta) d \theta=\eta_{n} / 2$.

Proof. Since $J_{\Phi_{n+1}}(\rho, \theta)=\left(\varphi_{n}(\theta) \mid \rho J_{\varphi_{n}}(\theta)\right)$, point (i) is evident.

Let us prove points (ii) and (iii) by induction on $n \geq 1$. If $n=1$, then (ii) and (iii) are immediate to verify. Let $n \geq 2$ and let $\theta=\left(\theta^{\prime}, \theta_{n}\right) \in I_{n-1} \times(0, \pi / 2)=I_{n}^{+}$. Define $c_{n}:=\cos \left(\theta_{n}\right), s_{n}:=\sin \left(\theta_{n}\right), \varphi_{n-1}^{\prime}:=\varphi_{n-1}\left(\theta^{\prime}\right)$ and $J_{n-1}^{\prime}:=J_{\varphi_{n-1}}\left(\theta^{\prime}\right)$. By definition, we have that $\varphi_{n}(\theta)=\left(c_{n} \varphi_{n-1}^{\prime}, s_{n}\right)^{T}$. In this way, it holds:

$$
J_{\varphi_{n}}(\theta)=\left(\begin{array}{c|c}
c_{n} J_{n-1}^{\prime} & -s_{n} \varphi_{n-1}^{\prime} \\
\hline 0 & c_{n}
\end{array}\right)
$$

and hence

$$
\left(\varphi_{n}(\theta)\right)^{T} J_{\varphi_{n}}(\theta)=\left(c_{n}^{2}\left(\varphi_{n-1}^{\prime}\right)^{T} J_{n-1}^{\prime} \mid-c_{n} s_{n}\left(\left(\varphi_{n-1}^{\prime}\right)^{T} \varphi_{n-1}^{\prime}-1\right)\right) .
$$


By induction, we know that $\left(\varphi_{n-1}^{\prime}\right)^{T} J_{n-1}^{\prime}=0$. On the other hand, $\varphi_{n-1}^{\prime}$ belongs to $S^{n-1}$ and hence $\left(\varphi_{n-1}^{\prime}\right)^{T} \varphi_{n-1}^{\prime}=1$. It follows that $\left(\varphi_{n}(\theta)\right)^{T} J_{\varphi_{n}}(\theta)=0$, as desired. This prove (ii). Observe that

$$
\begin{aligned}
\mathscr{I}_{n}(\theta) & =\operatorname{det}\left(J_{\Phi_{n+1}}(1, \theta)\right)=\operatorname{det}\left(\begin{array}{c|c|c|c}
c_{n} \varphi_{n-1}^{\prime} & c_{n} J_{n-1}^{\prime} & -s_{n} \varphi_{n-1}^{\prime} \\
\hline s_{n} & 0 & c_{n}
\end{array}\right)= \\
& =c_{n}^{n-1} \operatorname{det}\left(\begin{array}{c|c|c}
c_{n} \varphi_{n-1}^{\prime} & J_{n-1}^{\prime} & -s_{n} \varphi_{n-1}^{\prime} \\
\hline s_{n} & 0 & c_{n}
\end{array}\right) .
\end{aligned}
$$

The last determinant can be expanded w.r.t. the last row, to obtain

$$
\begin{aligned}
\mathscr{I}_{n}(\theta) & =c_{n}^{n-1}\left((-1)^{n} s_{n} \operatorname{det}\left(J_{n-1}^{\prime} \mid-s_{n} \varphi_{n-1}^{\prime}\right)+c_{n} \operatorname{det}\left(c_{n} \varphi_{n-1}^{\prime} \mid J_{n-1}^{\prime}\right)\right)= \\
& =c_{n}^{n-1}\left(s_{n}^{2} \operatorname{det}\left(\varphi_{n-1}^{\prime} \mid J_{n-1}^{\prime}\right)+c_{n}^{2} \operatorname{det}\left(\varphi_{n-1}^{\prime} \mid J_{n-1}^{\prime}\right)\right)=c_{n}^{n-1} \mathscr{I}_{n-1}\left(\theta^{\prime}\right) .
\end{aligned}
$$

Point (iii) follows by induction. Point (iv) is an immediate consequence of (iii) and the fact that the cosine is positive on $(-\pi / 2, \pi / 2)$.

It remains to prove (v) and (vi). Fix an integer $n \geq 1$ and $\theta \in I_{n}^{+}$. Bearing in mind (ii) and the equality $\left(\varphi_{n}(\theta)\right)^{T} \varphi_{n}(\theta)=1$, it follows that

$$
\left(J_{\Phi_{n+1}}(1, \theta)\right)^{T} J_{\Phi_{n+1}}(1, \theta)=\left(\begin{array}{c|c}
1 & 0 \\
\hline 0 & \left(J_{\varphi_{n}}(\theta)\right)^{T} J_{\varphi_{n}}(\theta)
\end{array}\right)
$$

and hence

$$
\mathscr{I}_{n}(\theta)^{2}=\operatorname{det}\left(\left(J_{\Phi_{n+1}}(1, \theta)\right)^{T} J_{\Phi_{n+1}}(1, \theta)\right)=\operatorname{det}\left(\left(J_{\varphi_{n}}(\theta)\right)^{T} J_{\varphi_{n}}(\theta)\right) .
$$

By combining this fact with (iv), we infer point (v). In particular, if $d \xi_{n}$ is the standard volume form on $S^{n}$, then $\left(\varphi_{n}\right)^{*}\left(d \xi_{n}\right)=\mathscr{I}_{n}(\theta) d \theta$ and hence (vi) holds:

$$
\int_{I_{n}^{+}} \mathscr{I}_{n}(\theta) d \theta=\frac{1}{2} \int_{S^{n}} d \xi_{n}=\frac{\eta_{n}}{2} .
$$

The proof is complete.

In what follows, we will use the notations fixed in the preceding section.

Proof of Lemma 2.2: Identify $\mathbb{R}^{m-1}$ with the vector subspace $\{0\} \times \mathbb{R}^{m-1}$ of $M \simeq$ $\mathbb{R}^{m}=\mathbb{R} \times \mathbb{R}^{m-1}$ and $S$ with the sphere $S^{m-2}$ in $\mathbb{R}^{m-1}$.

If $m=2$, then Lemma 2.2 is evident. Suppose $m \geq 3$ and fix $J \in S^{m-2}$. We will prove the following two inequalities

$$
\int_{\partial \Omega_{D}(S)}\left\|C_{S}(x, w)\right\| d \sigma_{w}<+\infty
$$

and

$$
\int_{\Omega_{D}(S)}\left\|C_{S}(x, w)\right\| d w<+\infty
$$


Evidently, points (6) and (7) are equivalent to Lemma 2.2. We organize the remainder of the proof into two steps. In the first, we prove (6). The second is devoted to the proof of (7).

Step I. For simplicity, we assume that $\partial D_{J}$ is connected. If this is not true, it suffices to consider each connected component of $\partial D_{J}$, suitably oriented.

Let $u=\alpha_{0}+\beta_{0} i \in \partial D$, let $v:=\alpha_{0}+\beta_{0} J \in \partial D_{J}$ and let $a, b:(0,1) \longrightarrow \mathbb{R}$ be $\mathscr{C}^{1}$-functions such that the map $(0,1) \ni t \mapsto a(t)+b(t) J \in \mathbb{C}_{J} \backslash\{v\}$ is a $\mathscr{C}^{1}$-embedding, which parametrizes $\partial D_{J} \backslash\{v\}$. Observe that $b(t)$ has at most two zeros in $(0,1)$. Define the $\mathscr{C}^{1}$-map $\Psi:(0,1) \times I_{m-2}^{+} \longrightarrow \mathbb{R}^{m}=\mathbb{R} \times \mathbb{R}^{m-1}$ and the dense open subset $\Xi$ of $\partial \Omega_{D}(S)$ by setting

$$
\Psi(t, \theta):=\left(a(t), b(t) \varphi_{m-2}(\theta)\right)^{T}
$$

and

$$
\Xi:=\left\{\alpha+\beta K \in \mathcal{Q}_{A} \mid \alpha, \beta \in \mathbb{R}, \alpha+\beta i \in \partial D \backslash\{u\}, K \in S_{+}^{m-2} \backslash\left(N \times \mathbb{R}^{m-3}\right)\right\} .
$$

It is immediate to see that the image of $\Psi$ coincides with $\Xi$ and the restriction $\psi$ : $(0,1) \times I_{m-2}^{+} \longrightarrow \Xi$ of $\Psi$ onto its image $\Xi$ is a $\mathscr{C}^{1}$-diffeomorphism.

Let $(t, \theta) \in(0,1) \times I_{m-2}^{+}$and let $J_{\Psi}(t, \theta)$ be the jacobian matrix of $\Psi$ at $(t, \theta)$. It holds:

$$
J_{\Psi}(t, \theta)=\left(\begin{array}{c|c}
a^{\prime}(t) & 0 \\
\hline b^{\prime}(t) \varphi_{m-2}(\theta) & b(t) J_{\varphi_{m-2}}(\theta)
\end{array}\right),
$$

where $a^{\prime}$ and $b^{\prime}$ denote the derivatives of $a$ and of $b$, respectively. By points (ii), (iv) and (v) of Lemma 3.1, we obtain:

$$
\left(J_{\Psi}(t, \theta)\right)^{T} J_{\Psi}(t, \theta)=\left(\begin{array}{c|c}
\left(a^{\prime}(t)\right)^{2}+\left(b^{\prime}(t)\right)^{2} & 0 \\
\hline 0 & (b(t))^{2}\left(J_{\varphi_{m-2}}(\theta)\right)^{T} J_{\varphi_{m-2}}(\theta)
\end{array}\right)
$$

and hence

$$
\sqrt{\operatorname{det}\left(\left(J_{\Psi}(t, \theta)\right)^{T} J_{\Psi}(t, \theta)\right)}=|b(t)|^{m-2} \mathscr{I}_{m-2}(\theta) \sqrt{\left(a^{\prime}(t)\right)^{2}+\left(b^{\prime}(t)\right)^{2}} .
$$

By combining point (iv) of Lemma 3.1 with the latter equality, we infer that

$$
\psi^{*}\left(d \sigma_{w}\right)=|b(t)|^{m-2} \mathscr{I}_{m-2}(\theta) \sqrt{\left(a^{\prime}(t)\right)^{2}+\left(b^{\prime}(t)\right)^{2}} d t d \theta .
$$

Bearing in mind (8) and performing the change of variable $w=\psi(t, \theta)$, we obtain:

$$
\begin{aligned}
& \int_{\partial \Omega_{D}(S)}\left\|C_{S}(x, w)\right\| d \sigma_{w}=\frac{2}{\eta_{m-2}} \int_{(0,1) \times I_{m-2}^{+}} \frac{\|C(x, w)\|}{|b(t)|^{m-2}} \psi^{*}\left(d \sigma_{w}\right)= \\
& =\frac{2}{\eta_{m-2}} \int_{(0,1) \times I_{m-2}^{+}}\|C(x, w)\| \mathscr{I}_{m-2}(\theta) \sqrt{\left(a^{\prime}(t)\right)^{2}+\left(b^{\prime}(t)\right)^{2}} d t d \theta .
\end{aligned}
$$

Since the integrand in the last integral is continuous, positive and bounded on $(0,1) \times$ $I_{m-2}^{+}$, inequality (6) holds. 
Step II. Let us prove (7). The proof is similar to the one of the preceding step, but slightly simpler. Let $(r, s)$ be the coordinates of $\mathbb{C} \simeq \mathbb{R}^{2}$. Define the $\mathscr{C}^{1}$-map $\Gamma:(D \backslash \mathbb{R}) \times I_{m-2}^{+} \longrightarrow \mathbb{R}^{m}=\mathbb{R} \times \mathbb{R}^{m-1}$ and the dense open subset $\Upsilon$ of $\Omega_{D}(S) \backslash \mathbb{R}$ by setting

$$
\Gamma(r, s, \theta):=\left(r, \Phi_{m-1}(s, \theta)\right)^{T}
$$

and

$$
\Upsilon:=\left\{r+s K \in \mathcal{Q}_{A} \mid r, s \in \mathbb{R}, r+s i \in D \backslash \mathbb{R}, K \in S_{+}^{m-2} \backslash\left(N \times \mathbb{R}^{m-3}\right)\right\} .
$$

It is easy to verify that the image of $\Gamma$ is equal to $\Upsilon$ and the restriction $\gamma:(D \backslash \mathbb{R}) \times$ $I_{m-2}^{+} \longrightarrow \Upsilon$ of $\Gamma$ onto its image $\Upsilon$ is a $\mathscr{C}^{1}$-diffeomorphism.

Given $(r, s, \theta) \in(D \backslash \mathbb{R}) \times I_{m-2}^{+}$, it is immediate to see that the determinant $\operatorname{det}\left(J_{\Gamma}(r, s, \theta)\right)$ of the jacobian matrix of $\Gamma$ at $(r, s, \theta)$ is equal to $\operatorname{det}\left(J_{\Phi_{m-1}}(s, \theta)\right)$ and hence, by point (i) of Lemma 3.1, we have:

$$
\left|\operatorname{det}\left(J_{\Gamma}(r, s, \theta)\right)\right|=|s|^{m-2} \mathscr{I}_{m-2}(\theta) .
$$

Using the change of variable $w=\Gamma(r, s, \theta)$ and (9), we obtain:

$$
\begin{aligned}
\int_{\Omega_{D}(S)}\left\|C_{S}(x, w)\right\| d w & =\frac{2}{\eta_{m-2}} \int_{(D \backslash \mathbb{R}) \times I_{m-2}^{+}} \frac{\|C(x, w)\|}{|s|^{m-2}}|s|^{m-2} \mathscr{I}_{m-2}(\theta) d r d s d \theta= \\
& =\frac{2}{\eta_{m-2}} \int_{D \times I_{m-2}^{+}}\|C(x, w)\| \mathscr{I}_{m-2}(\theta) d r d s d \theta
\end{aligned}
$$

This equality implies immediately (7).

Proof of Theorem 2.3: During this proof, we will use the maps $\psi$ and $\Gamma$ defined in the preceding one. If $m=2$, then, as we have just said, Theorem 2.3 is equivalent to Theorem 27 of [4]. Suppose $m \geq 3$. Let us prove that, fixed any $J \in S$, the following equality holds:

$$
\begin{array}{r}
\int_{\partial \Omega_{D}(S)} C_{S}(x, w) \mathbf{n}(w) f(w) d \sigma_{w}-2 \int_{\Omega_{D}(S)} C_{S}(x, w) \frac{\partial f}{\partial x^{c}}(w) d w= \\
=\int_{\partial D_{J}} C(x, y) J^{-1} d y f(y)-\int_{D_{J}} C(x, y) J^{-1} d y^{c} \wedge d y \frac{\partial f}{\partial x^{c}}(y) .
\end{array}
$$

Bearing in mind Theorem 27 of [4], equation (10) is equivalent to Theorem 2.3. Observe that, if $(t, \theta) \in(0,1) \times I_{m-2}^{+}$and $w=\psi(t, \theta)$, then

$$
\mathbf{n}(w)=\frac{b^{\prime}(t)-a^{\prime}(t) J_{\theta}}{\sqrt{\left(a^{\prime}(t)\right)^{2}+\left(b^{\prime}(t)\right)^{2}}},
$$

where $J_{\theta}:=\varphi_{m-2}(\theta) \in S$. Making use of (8), (11) and of the change of variable 
$w=\psi(t, \theta)$, we obtain:

$$
\begin{aligned}
& \frac{\eta_{m-2}}{2} \int_{\partial \Omega_{D}(S)} C_{S}(x, w) \mathbf{n}(w) f(w) d \sigma_{w}= \\
& =\int_{(0,1) \times I_{m-2}^{+}} C(x, w)\left(b^{\prime}(t)-a^{\prime}(t) J_{\theta}\right) \mathscr{I}_{m-2}(\theta) d t d \theta f(w)= \\
& =\int_{I_{m-2}^{+}} \mathscr{I}_{m-2}(\theta) d \theta \int_{0}^{1} C(x, w)\left(b^{\prime}(t)-a^{\prime}(t) J_{\theta}\right) d t f(w)= \\
& =\int_{I_{m-2}^{+}} \mathscr{I}_{m-2}(\theta) d \theta \int_{0}^{1} C(x, w) J_{\theta}^{-1} d\left(a(t)+b(t) J_{\theta}\right) f(w),
\end{aligned}
$$

since $J_{\theta}^{-1}=-J_{\theta}$. Using the change of variable $w=\Gamma(r, s, \theta)=\left(r, s J_{\theta}\right)$ and (9) again, we also have:

$$
\begin{aligned}
& \eta_{m-2} \int_{\Omega_{D}(S)} C_{S}(x, w) \frac{\partial f}{\partial x^{c}}(w) d w=\int_{D \times I_{m-2}^{+}} C(x, w) \mathscr{I}_{m-2}(\theta) 2 d r d s d \theta \frac{\partial f}{\partial x^{c}}(w)= \\
& =\int_{I_{m-2}^{+}} \mathscr{I}_{m-2}(\theta) d \theta \int_{D_{J_{\theta}}} C(x, y) J_{\theta}^{-1} d y^{c} \wedge d y \frac{\partial f}{\partial x^{c}}(y)
\end{aligned}
$$

where $2 d r d s=J_{\theta}^{-1} d y^{c} \wedge d y$ if $y=r+s J_{\theta} \in D_{J_{\theta}}$.

From (12) and (13), we get

$$
\begin{array}{r}
\frac{\eta_{m-2}}{2} \int_{\partial \Omega_{D}(S)} C_{S}(x, w) \mathbf{n}(w) f(w) d \sigma_{w}-\eta_{m-2} \int_{\Omega_{D}(S)} C_{S}(x, w) \frac{\partial f}{\partial x^{c}}(w) d w= \\
=\int_{I_{m-2}^{+}} \mathscr{I}_{m-2}(\theta) d \theta\left(\int_{0}^{1} C(x, w) J_{\theta}^{-1} d\left(a(t)+b(t) J_{\theta}\right) f(w)-\right. \\
\left.-\int_{D_{J_{\theta}}} C(x, y) J_{\theta}^{-1} d y^{c} \wedge d y \frac{\partial f}{\partial x^{c}}(y)\right) .
\end{array}
$$

The Cauchy formula proved in Theorem 27 of [4] gives that

$$
\begin{aligned}
& \int_{0}^{1} C(x, w) J_{\theta}^{-1} d\left(a(t)+b(t) J_{\theta}\right) f(w)-\int_{D_{J_{\theta}}} C(x, y) J_{\theta}^{-1} d y^{c} \wedge d y \frac{\partial f}{\partial x^{c}}(y)= \\
& =\int_{\partial D_{J}} C(x, y) J^{-1} d y f(y)-\int_{D_{J}} C(x, y) J^{-1} d y^{c} \wedge d y \frac{\partial f}{\partial x^{c}}(y)=2 \pi f(x)
\end{aligned}
$$

for each $\theta \in I_{m-2}^{+}$. Therefore, thanks to point (vi) of Lemma 3.1, we have:

$$
\begin{aligned}
& \frac{\eta_{m-2}}{2} \int_{\partial \Omega_{D}(S)} C_{S}(x, w) \mathbf{n}(w) f(w) d \sigma_{w}-\eta_{m-2} \int_{\Omega_{D}(S)} C_{S}(x, w) \frac{\partial f}{\partial x^{c}}(w) d w= \\
& =\frac{\eta_{m-2}}{2}\left(\int_{\partial D_{J}} C(x, y) J^{-1} d y f(y)-\int_{D_{J}} C(x, y) J^{-1} d y^{c} \wedge d y \frac{\partial f}{\partial x^{c}}(y)\right)= \\
& =\int_{I_{m-2}^{+}} \mathscr{I}_{m-2}(\theta) d \theta(2 \pi f(x))=\pi \eta_{m-2} f(x) .
\end{aligned}
$$


Equality (10) is proved and the proof is complete.

Proof of Theorem 2.4: Let $f \in \mathcal{S}^{1}\left(\partial \Omega_{D}(S), A\right)$. For each $\theta \in I_{m-2}^{+}$, let $J_{\theta}=$ $\varphi_{m-2}(\theta) \in S$ be as in the preceding proof. Denote by $F_{\theta}^{+}$and $F_{\theta}^{-}$the Cauchy-type integrals defined on $D_{J_{\theta}}$ and $\mathbb{C}_{J_{\theta}} \backslash \bar{D}_{J_{\theta}}$ by

$$
F_{\theta}^{ \pm}(x):=\frac{1}{2 \pi} \int_{\partial D_{J_{\theta}}} C(x, w) J_{\theta}^{-1} d w f(w) .
$$

Since the restriction $f_{\mid \partial D_{J_{\theta}}}$ is of class $\mathscr{C}^{1}, F_{\theta}^{+}$extends continuously to $\bar{D}_{J_{\theta}}$ and $F_{\theta}^{-}$ extends as a continuous function on $\mathbb{C}_{J_{\theta}} \backslash D_{J_{\theta}}$. Moreover, the classical Sokhotskiı̌-Plemelj formula (see $[10, \S 2])$ holds:

$$
F_{\theta}^{+}(x)-F_{\theta}^{-}(x)=f(x) \text { for each } x \in \partial D_{J_{\theta}} .
$$

The functions $F_{\theta}^{+}$and $F_{\theta}^{-}$are slice regular on $\Omega_{D}$ and $\mathcal{Q}_{A} \backslash \bar{\Omega}_{D}$, respectively. The continuity of their restrictions to $\mathbb{C}_{J_{\theta}}$ up to the boundary implies the continuity of the inducing stem functions (see the proof of Proposition 5 of [4]). In view of Proposition 7(1) of [4], also the functions $F_{\theta}^{ \pm}$are continuous up to the boundary. Given a point $\hat{x}=\alpha+\beta I \in \partial \Omega_{D}(S)$, let $x^{\prime}=\alpha+\beta J_{\theta}$ and $x^{\prime \prime}=\alpha-\beta J_{\theta}$. From (15), we get: $f\left(x^{\prime}\right)=F_{\theta}^{+}\left(x^{\prime}\right)-F_{\theta}^{-}\left(x^{\prime}\right)$ and $f\left(x^{\prime \prime}\right)=F_{\theta}^{+}\left(x^{\prime \prime}\right)-F_{\theta}^{-}\left(x^{\prime \prime}\right)$. The representation formula (see [4, Prop. 6]) applied to $f$ and to $F_{\theta}^{ \pm}$gives

$$
f(\hat{x})=\frac{1}{2}\left(f\left(x^{\prime}\right)+f\left(x^{\prime \prime}\right)\right)-\frac{I J_{\theta}}{2}\left(f\left(x^{\prime}\right)-f\left(x^{\prime \prime}\right)\right)=F_{\theta}^{+}(\hat{x})-F_{\theta}^{-}(\hat{x}) .
$$

From (12) and (14), we get:

$$
\begin{aligned}
F_{S}^{ \pm}(x) & =\frac{1}{2 \pi} \int_{\partial \Omega_{D}(S)} C_{S}(x, w) \mathbf{n}(w) f(w) d \sigma_{w}= \\
& =\frac{1}{\pi \eta_{m-2}} \int_{I_{m-2}^{+}} \mathscr{I}_{m-2}(\theta) d \theta \int_{0}^{1} C(x, w) J_{\theta}^{-1} d\left(a(t)+b(t) J_{\theta}\right) f(w)= \\
& =\frac{2}{\eta_{m-2}} \int_{I_{m-2}^{+}} \mathscr{I}_{m-2}(\theta) d \theta F_{\theta}^{ \pm}(x) .
\end{aligned}
$$

Let $U$ be a compact neighborhood of $\partial D$, invariant w.r.t. complex conjugation. There exists a positive constant $K$ such that $\left\|F_{\theta}^{+}(x)\right\| \leq K$ for each $x \in \bar{D}_{J_{\theta}}$ and $\left\|F_{\theta}^{-}(x)\right\| \leq K$ for each $x \in\left(\mathbb{C}_{J_{\theta}} \backslash D_{J_{\theta}}\right) \cap \Omega_{U}$. The representation formula gives $\left\|F_{\theta}^{+}(x)\right\| \leq 2 K$ for each $x \in \bar{\Omega}_{D}$ and $\left\|F_{\theta}^{-}(x)\right\| \leq 2 K$ for each $x \in\left(\mathcal{Q}_{A} \backslash \Omega_{D}\right) \cap \Omega_{U}$. Then Lebesgue's dominated convergence theorem ensures that $F_{S}^{ \pm}$extend continuously up to the boundary $\partial \Omega_{D}$. Moreover, from (16) and (17), we get the jump $F_{S}^{+}(\hat{x})-F_{S}^{-}(\hat{x})$ at $\hat{x} \in \partial \Omega_{D}(S)$ :

$$
\begin{aligned}
F_{S}^{+}(\hat{x})-F_{S}^{-}(\hat{x}) & =\frac{2}{\eta_{m-2}} \int_{I_{m-2}^{+}} \mathscr{I}_{m-2}(\theta) d \theta\left(F_{\theta}^{+}(\hat{x})-F_{\theta}^{-}(\hat{x})\right)= \\
& =\frac{2}{\eta_{m-2}} \int_{I_{m-2}^{+}} \mathscr{I}_{m-2}(\theta) d \theta f(\hat{x})=f(\hat{x}) .
\end{aligned}
$$




\section{References}

[1] G. Gentili and D.C. Struppa, A new approach to Cullen-regular functions of a quaternionic variable, C. R. Math. Acad. Sci. Paris 342 (2006), pp. 741-744.

[2] - A new theory of regular functions of a quaternionic variable, Adv. Math. 216 (2007), pp. 279-301.

[3] F. Colombo, I. Sabadini, and D.C. Struppa, Slice monogenic functions, Israel J. Math. 171 (2009), pp. 385-403.

[4] R. Ghiloni and A. Perotti, Slice regular functions on real alternative algebras, Adv. Math. 226 (2011), pp. 1662-1691.

[5] - A new approach to slice regularity on real algebras, in Hypercomplex analysis and its Applications, Birkhäuser, Basel, 2011, pp. 109-124.

[6] F. Colombo, G. Gentili, and I. Sabadini, A Cauchy kernel for slice regular functions, Ann. Global Anal. Geom. 37 (2010), pp. 361-378.

[7] F. Colombo and I. Sabadini, The Cauchy formula with s-monogenic kernel and a functional calculus for noncommuting operators, J. Math. Anal. Appl. 373 (2011), pp. 655-679.

[8] - A structure formula for slice monogenic functions and some of its consequences, in Hypercomplex analysis, Birkhäuser, Basel, 2009, pp. 101-114.

[9] F. Colombo, I. Sabadini, and D.C. Struppa, The Pompeiu formula for slice hyperholomorphic functions, Michigan Math. J. 60 (2011), pp. 163-170.

[10] A.M. Kytmanov, The Bochner-Martinelli integral and its applications, Birkhäuser Verlag, Basel, 1995. 